\title{
Endovascular Treatment of Wide-necked Cerebral Aneurysms with an Acute Angle Branch Incorporated into the Sac: Novel methods of Branch Access in 8 Aneurysms
}

\author{
Bae Ju Kwon, MD, PhD', Dae-Hee Seo, MD², Young-Soo Ha, MD³, Kyu-Chang Lee, MD²
}

Purpose: The optimal management of geometrically complex aneurysms remains challenging. The aim of this retrospective study was to evaluate the safety and feasibility of branch-selective technique (BT) in wide-necked aneurysms with an acute angle branch incorporated into the sac.

Materials and Methods: Eight consecutive patients harboring wide-necked cerebral aneurysms with an incorporated, acute angle branch (mean, 30.4 ) underwent coiling over an 18-month period. Dome-to-neck ratio ranged from 0.9 to 1.8 (mean, 1.2). Every procedure utilized BT, i.e., stent- or catheter-assisted coiling through the incorporated branch.

Results: Technical success was achieved in all cases. With the aim to avoid the risk of aneurysmal rupture during struggling intraaneurysmal wire navigation, a 'looping method' and retrograde approach of a preshaped 0.014' microcatheter ( $C$ or $J$ ) was used for branch access in five cases and a 'looping method' and antegrade approach in one case. In the remaining one, just the C-preshape was enough to directly enter the branch without intraaneurysmal wire navigation. Overall, stent-assisted coiling was performed in seven cases, while catheter-assisted coiling was undertaken in one. The only complication was thrombotic posterior inferior cerebellar artery occlusion in one case, which was recanalized after tirofiban infusion. New neurological deficits were not identified in any cases.

Conclusion: BT seems safe and feasible for wide-necked aneurysms with an acute angle branch incorporated into the sac. The looping method may offer safe access to the incorporated, acute angle branch and should be considered for replacement of the fearful intra-aneurysmal wire navigation.

Key Words : Endovascular procedures; Intracranial aneurysm; Branch-selective technique

'Department of Radiology and ${ }^{2}$ Neurosurgery, Kwandong University Myongji Hospital, Goyang, Korea

${ }^{3}$ Department of Neurosurgery, Jecheon Myongji Hospital, Jecheon, Korea

Received July 9, 2012; accepted after revision August 7, 2012.

Correspondence to: Bae Ju Kwon, MD, PhD, Neurovascular Center, Myongji Hospital, 697-24 Hwajeong-dong, Deogyang-gu, Goyang-si, Gyeonggi-do 412-270, Korea.

Tel. 82.31.810.5599 Fax.82.31.810.7171 E-mail: bjkwon74@gmail.com

This is an Open Access article distributed under the terms of the Creative Commons Attribution Non-Commercial License (http://creativecommons.org/licenses/by-nc/3.0) which permits unrestricted non-commercial use, distribution, and reproduction in any medium, provided the original work is properly cited. 
Since the International Subarachnoid Aneurysm Trial, endovascular treatment gained ground in the management of cerebral aneurysms [1]. Currently, coiling largely supersedes clipping in the treatment of cerebral aneurysms and, with the rapid development of devices and techniques, the field of neurovascular intervention is likely to expand further. However, the lower durability of coiling has yet to be solved.

Complex and difficult aneurysms for coiling refer to aneurysms that have a wide neck, very small diameter, bizarre shape (e.g., multilobulation, tube-like elongation, or bent shape), anatomical complexity precipitating the microcatheter kickback, branch incorporation into the sac, and so on. If an incorporated branch supplies the pivotal area of the brain, every effort should be made to preserve that branch during the procedures. However in most cases, difficulties are encountered in the superselection of the incorporated branch and is frequently weighted as a result of acute angle branching. Moreover, aggressive intra-aneurysmal wire navigation may lead to catastrophic aneurysm perforation. If no pre-specified method for preserving the branch is planned beforehand, the procedure may be aborted or finished with complications. If a nonbranch-selective technique (NT), such as multi-catheter or balloon remodeling technique, or stent-assisted technique that places a stent in the parent artery, may result in significant branch compromise and thrombosis, it would be very hard to recanalize the occluded branch because of excessive difficulty in branch access. Particularly in balloon remodeling technique [2], as a balloon catheter system is placed in the parent artery across the aneurysm neck, overinflation and intraaneurysmal herniation of the compliant balloon are necessary for protection of the incorporated branch and its remodeling capability can be limited when the incorporated branch orifice is poorly covered. As for branch-selective technique (BT), including both stentassisted coiling and catheter-assisted technique through the incorporated branch, an excessively shaped microguidewire usually does not meet the required angle for accessing the incorporated, acute angle branch and easily passes the branch orifice toward the aneurysm dome. However, aggressive wire navigation for branch access should be restricted to avoid the risk of aneurysm perforation. Therefore, for the purpose of preserving the incorporated branch, NT requires a sustainable smaller coil frame to leave enough room in the aneurysm neck toward incorporated branch, whereas BT necessitates special methods of wire and microcatheter manipulations to safely superselect the incorporated, acute angle branch.

Recently, our institution has set up a biplane system with three-dimensional imaging capability for endovascular procedures, and in case of wide-necked and branch-incorporated aneurysm, we have made efforts at finding how to smoothly access the incorporated branch on a case-by-case basis. We sought to evaluate the technical success rate, complications, and new neurological deficits of the BT procedures.

\section{MATERIALS AND METHODS}

This retrospective study was approved by the institutional review board, and informed consent was waived. One hundred twenty-seven patients with 135 cerebral aneurysms underwent endovascular treatment between September 2010 and February 2012 at our institution. Our database was searched for all cases of wide-necked aneurysms with an acute angle branch incorporated into the sac. A wide-necked aneurysm was defined as having a neck size of $\geq 4 \mathrm{~mm}$ or a dome-to-neck ratio of $<2$, while "an acute angle branch incorporated into the sac" was as a branch arising from the sac at an angle of less than $90^{\circ}$ relative to the parent artery. Patients who had undergone Y-configuration stenting were excluded from the study because it needed different approaches and complicated processes with additional procedural risks. Eight $(6 \%)$ cases were found to meet the above conditions and their medical records were reviewed. There were five women and three men, with a mean age of 57 years (range, $43-69$ years). Two patients presented with subarachnoid hemorrhage respectively graded as 2 and 3 by Hunt and Hess scale and six were asymptomatic. Six patients underwent primary embolization and two received repeat embolization for recanalized aneurysms. Three aneurysms were found at the posterior inferior cerebellar artery (PICA) origin, two at the middle cerebral artery (MCA) bifurcation, two at the basilar bifurcation, and one at the posterior communicating artery-internal carotid artery (Pcom-ICA). The mean values of aneurysm size, dome-to-neck ratio, and incorporated branch angle were $7.8 \mathrm{~mm}$ (range, 4.3 to $13.1 \mathrm{~mm}$ ), 1.2 $\mathrm{mm}$ (range, 0.9 to $1.8 \mathrm{~mm}$ ), and $30.4^{\circ}$ (range, -20 to $80^{\circ}$ ), respectively. Clinical and anatomical data are summarized in Table 1.

\section{Procedure}

All patients underwent cerebral angiography with three dimensional image reconstruction using the Innova $3131^{\text {IQ }}$ biplane system (GE Healthcare 
Wide-necked Aneurysms with an Incorporated Acute Angle Branch

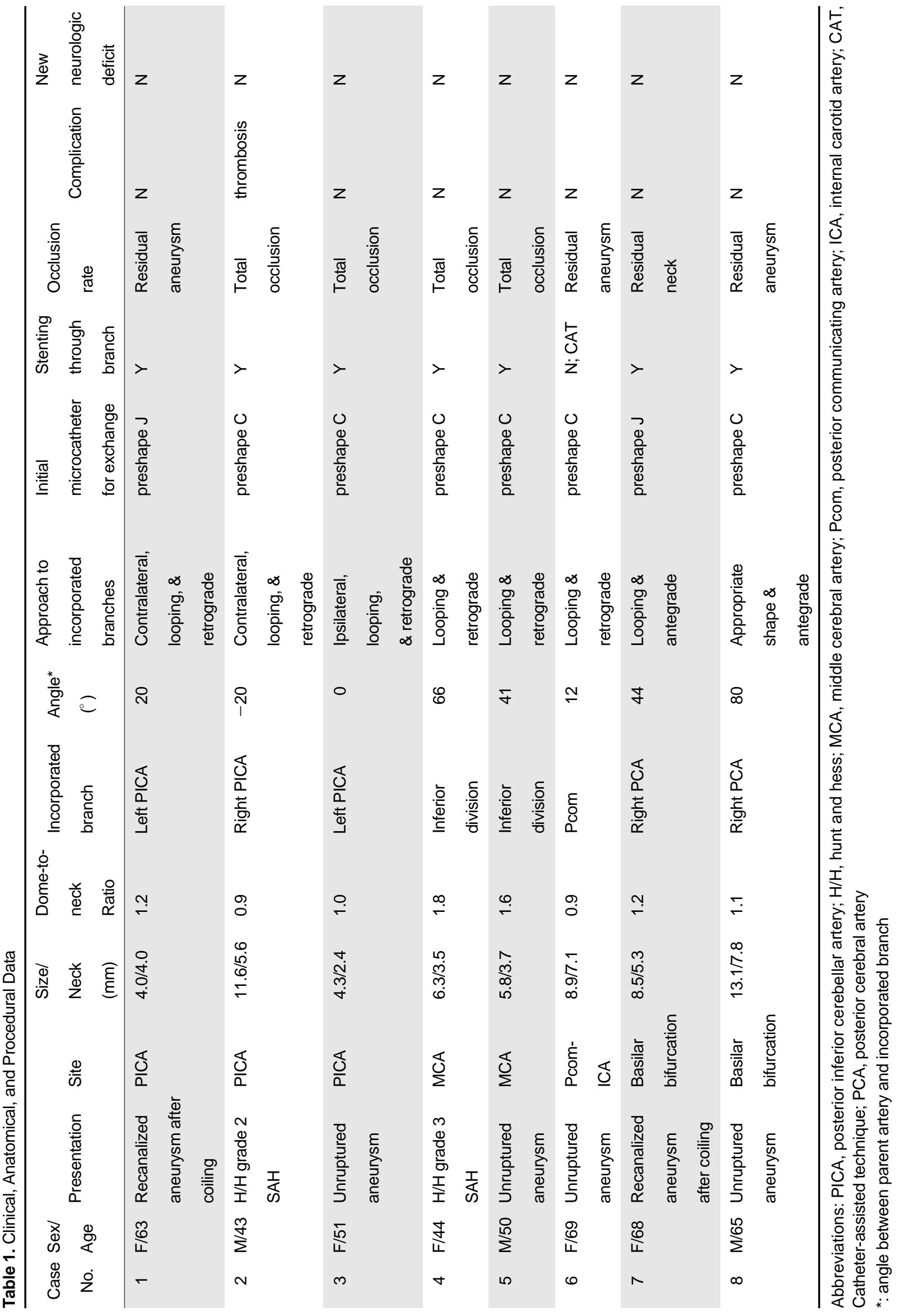




\section{Bae Ju Kwon, et al.}

Technologies, Waukesha, WI, USA). After reviewing these images, a group of neurosurgeons and neuroradiologists discussed any different opinions and reached a consensus for treatment of coiling. The use of an endovascular technique, BT versus NT, was left to the discretion of the operator, who took into account the

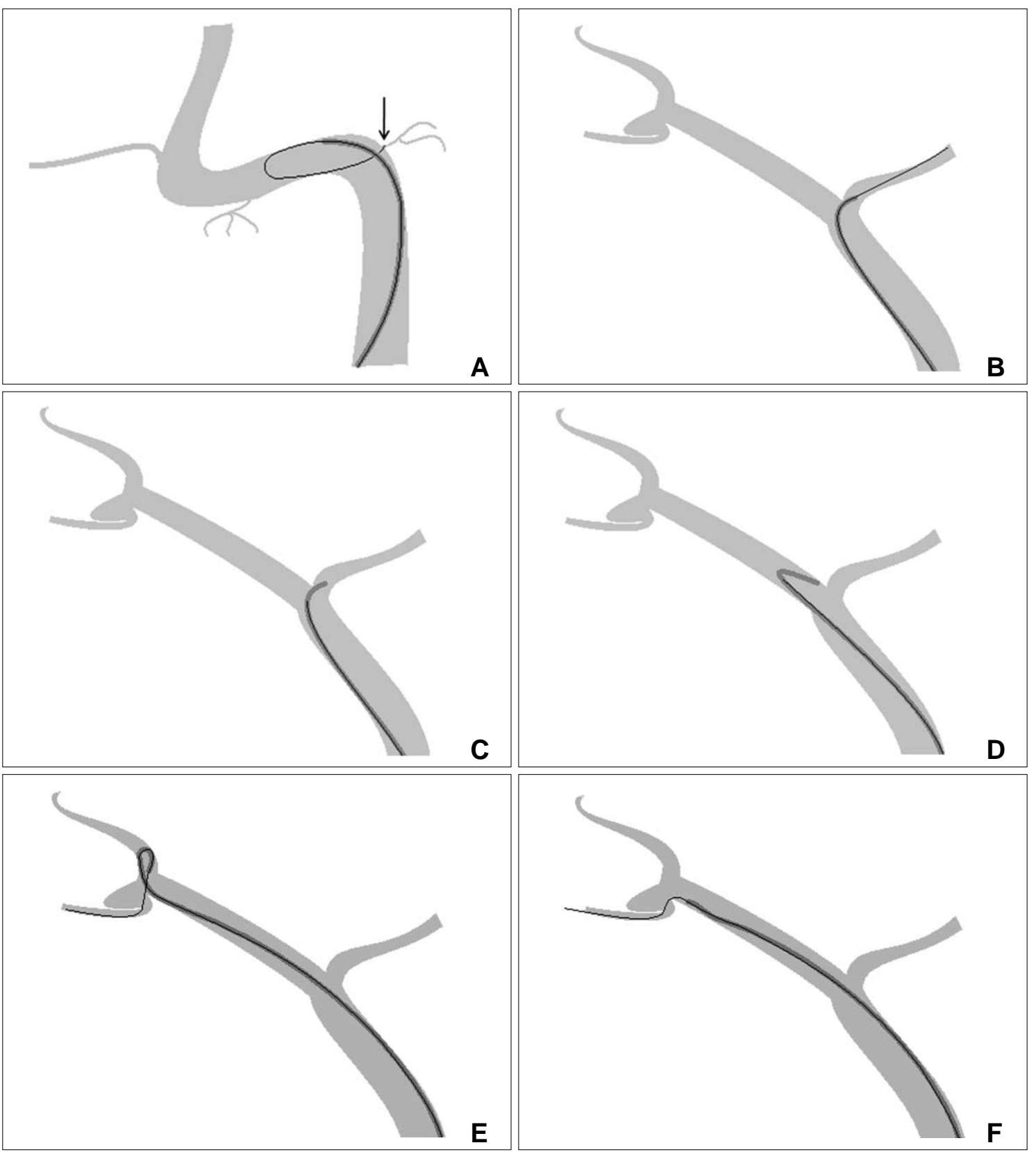

Fig. 1. Schematic illustration of looping methods in the anterior circulation.

Microcatheter looping is well completed in the cavernous segment and terminus of the ICA. When the wire tip is checked by the orifice of a small branch, such as meningohypophyseal artery (black arrow), gentle push of the wire can make the looping of the wire in the cavernous ICA (A). Advance of the microcatheter over the wire then is necessary for microcatheter looping, which can be used for coiling of Pcom-ICA aneurysms. If a targeted aneurysm is located at the MCA bifurcation, an access to the incorporated branch requires a series of steps at the ICA terminus and MCA bifurcation: 1) the C or J-preshaped microcatheter tip is slightly advanced into the A1 segment over the wire $(\mathbf{B}), 2)$ the wire is withdrawn into the microcatheter in the proximal half of the $\mathrm{C}$ or $\mathrm{J}$ curve (C), 3) gentle push of the microcatheter makes it looped in the M1 segment because the empty distal half of the curve is prone to bend in this relatively straightforward and larger segment (D), 4) after advancing and placing this looped microcatheter in the gently curved parent artery past the aneurysm, the wire can come out of the microcatheter and access the incorporated branch retrograde via a new smooth track (E), 5) Once the wire enters the incorporated branch, it should be placed in a far distal branch. The looped portion then should be straightened before advance over the wire (F). According to the technique, stent-assisted coiling vs. catheter-assisted coiling, the need for exchanging microcatheters is determined. 
degree of branch incorporation, neck size, dome-toneck ratio, and the anatomical relationship among parent arteries, aneurysms, and branches, etc. All procedures were carried out under general anesthesia. After femoral sheath placement, systemic heparinization was started with a loading dose of 3,000 IU and was maintained with a dose of 1,000 IU on an hourly basis. For two patients with a ruptured aneurysm, the same regimen of systemic heparinization was followed after the first or second coil placement. If NT, as a planned technique, failed to make an appropriate coil frame not to compromise incorporated branches, the technique was switched to BT. For BT, the steps for branch access were all initiated by the introduction of a $0.014^{\prime} \mathrm{C}$ - or J-preshaped microcatheter, because it had some advantages over a $0.021^{\prime}$ stent delivery catheter, including an acute angle tip with easy shapability and less traumatic manipulation in the cerebral artery required for branch access. Intraaneurysmal antegrade wire navigation was discarded in branch access because of the risk of aneurysmal perforation; instead a special method to exaggerate the microcatheter curve, 'looping', was carried out. The looped catheter was created in the large-caliber proximal artery, such as ICA or vertebral artery (VA), by two methods: 1) intraluminal wire looping followed by catheter looping (Fig. 1A); 2) a resistant-branch method using a even smaller branch as a resistant vessel for advance, such as A1 segment at the ICA bifurcation or $\mathrm{C} 2$ or $\mathrm{C} 3$ branch at the cervical VA (Fig. 1B - D). According to the
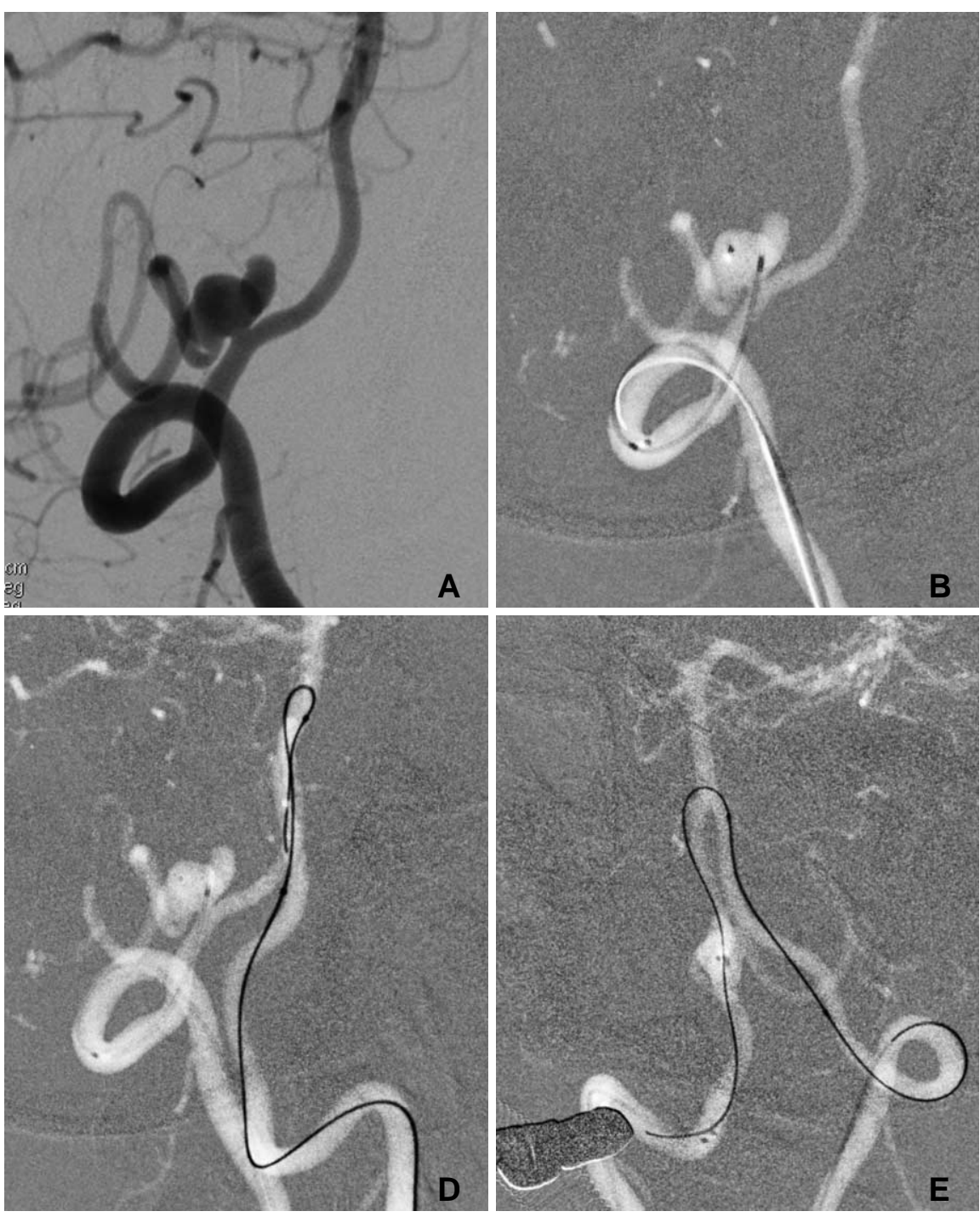

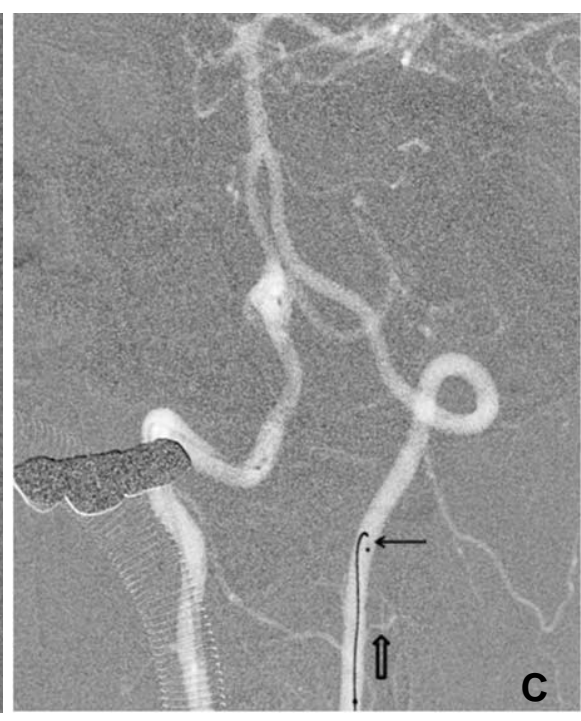

Fig. 2. A 43-year-old male with a ruptured aneurysm incorporating the right PICA (Case 2).

A. Working projection shows right PICA aneurysm with PICA incorporation. The PICA arises from the sac at an angle of $-20^{\circ}$ relative to the VA.

B. Two microcatheters via the ipsilateral VA are shown in the aneurysm for dual-catheter technique in combination with branchselective stent-assisted coiling.

C. Roadmap image shows a looped microcatheter (black arrow) in the contralateral VA for the purpose of crossing the acute angle vertebral-vertebral junction and retrograde approach of the right PICA. The loop can be made in the posterior circulation through $\mathrm{C} 2$ or $\mathrm{C} 3$ branch like the $\mathrm{A} 1$ segment of the anterior circulation (open arrow).

D, E. Roadmap image shows that the wire is out of the looped microcatheter in the basilar artery and placed far downward in the ipsilateral VA.

Continued 

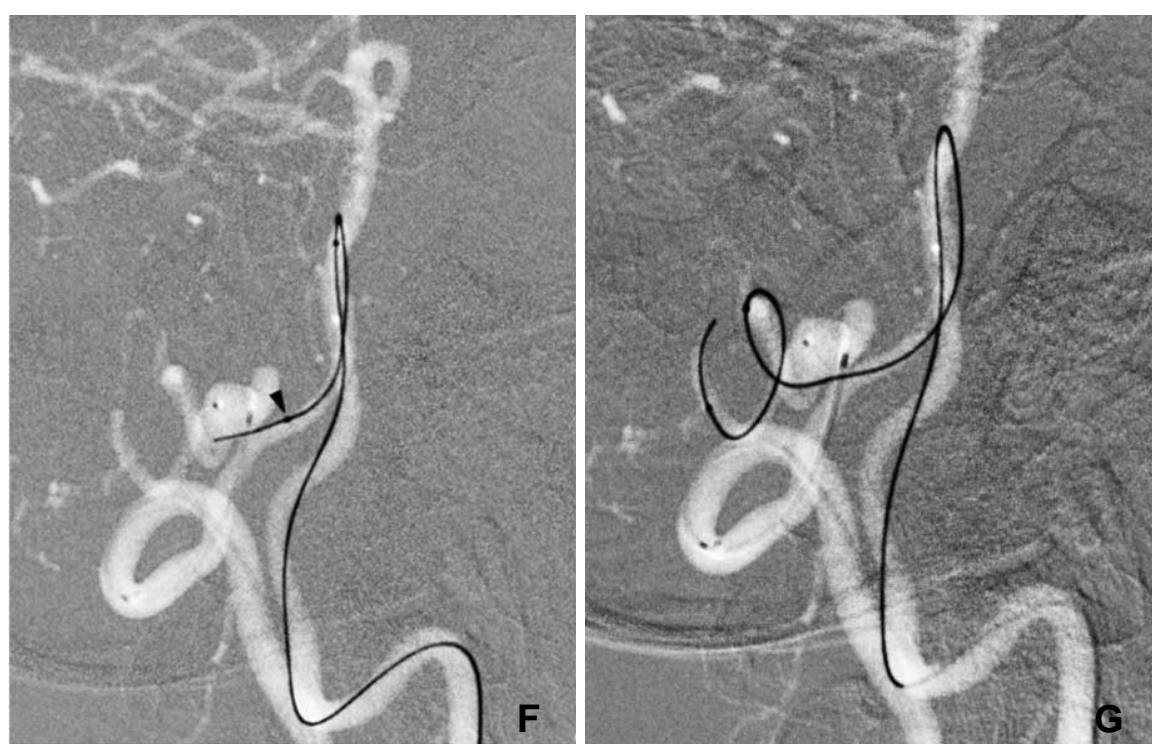

Fig. 2. F. Once the microcatheter is advanced to the PICA orifice over the wire (black arrowhead), the wire can access the PICA via a new retrograde smooth track.

G. Roadmap image shows the microcatheter over the wire in the PICA. It should be exchanged for a stent delivery catheter for stent-assisted coiling.

H. Subtraction angiogram obtained after the eighth coil placement reveals occluded PICA (white arrows).

I. Completion angiogram obtained after intraarterial tirofiban injection $(0.5 \mathrm{mg})$ shows the restored flow but some tiny clots at the proximal PICA (open arrowheads). The stent markers are barely identifiable due to digital subtraction (dotted line).
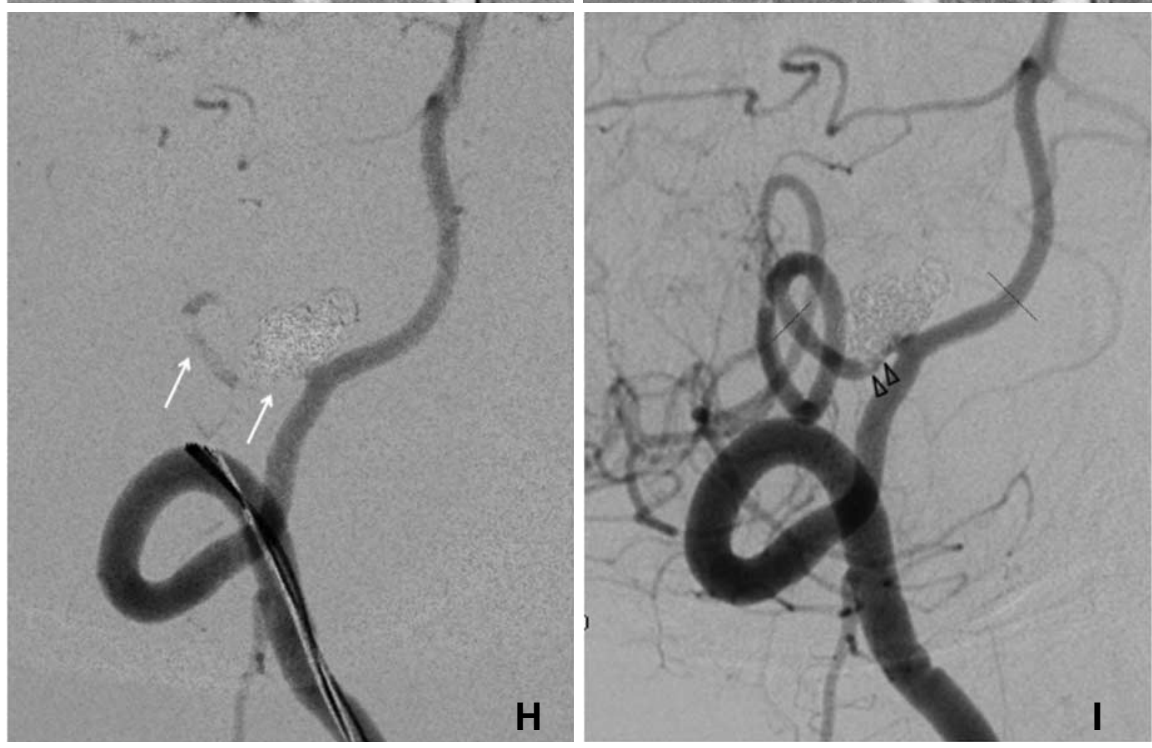

anatomic relationship among the parent artery, aneurysm, and branch, the looped microcatheter could directly approach the incorporated branch; otherwise further steps were necessary around the aneurysm (Fig. $1 \mathrm{E}, \mathrm{F})$. The microcatheter then was exchanged for a larger stent delivery catheter or used for branch protection according to a planned technique, stent vs. catheter assisted coiling.

For patients with unruptured aneurysms, aspirin (100 $\mathrm{mg}$ /day) and clopidogrel ( $75 \mathrm{mg}$ /day) were administered at least three days before the procedure. Postprocedure medication following stent-assisted coiling included life-long aspirin $(100 \mathrm{mg} /$ day $)$ and clopidogrel (75 mg/day) administration for a 3-month period. Patients with a ruptured aneurysm were given a loading dose of aspirin $(100 \mathrm{mg})$ and clopidogreal (300 $\mathrm{mg}$ ) via a Levin tube just after procedures, and were placed on the same postprocedure medication regime.

\section{Immediate outcome}

Any kind of procedural complications were listed. Technical success was defined as a greater than $90 \%$ aneurysm occlusion, as demonstrated by completion angiography. The occlusion rate was classified as 'total occlusion (no contrast filling in the aneurysm but the incorporated branch orifice)', 'residual neck' (small contrast filling only in the neck other than the incorporated branch orifice), 'residual aneurysm' (contrast filling in the body). Immediate clinical outcome signified new neurological deficits until discharge postprocedure. 


\section{RESULTS}

Technical success was achieved in all eight $(100 \%)$ cases. The looping method was used to perform $\mathrm{BT}$ in seven cases: 3 PICA aneurysms, 2 MCA aneurysms, 1 Pcom-ICA aneurysm, and one recanalized basilar bifurcation aneurysm after coiling. Three cases with a PICA aneurysm had extremely acute angles between the VA and PICA $\left(20^{\circ},-20^{\circ}\right.$, and $\left.0^{\circ}\right)$ and needed to be approached retrograde with catheter looping, which was made by a resistant-branch method at the upper cervical VA. If the contralateral VA was large enough to keep a guiding catheter, the PICA was accessed retrograde through it (Fig. 2). Catheter looping was also required to cross over the acute angle vertebrovertebral junction. Further steps were the same as illustrations in Fig. 1E and 1F. In Case 2 with a ruptured PICA aneurysm, thrombus occurred in the aneurysm neck during the last coil packing and it caused occlusion of the PICA (Fig. 2H). Tirofiban (0.5 $\mathrm{mg}$ in total) was infused through the microcatheter and restored the PICA flow (Fig. 2I). Additionally, intravenous infusion was continued during the following 12 hours $(0.1 \mu \mathrm{g} / \mathrm{kg} / \mathrm{min})$. For two MCA bifurcation aneurysms with an incorporated, acute angle branch $\left(66^{\circ}\right.$ and $\left.41^{\circ}\right)$, a C-preshaped microcatheter was used to superselect the incorporated branch by the looping method and retrograde approach (Fig. 1B - F and Fig. 3). For Case 6 with a Pcom-ICA aneurysm, the same method and approach was adopted to perform catheter-assisted coiling for Pcom-protective embolization. Repeat coiling for basilar bifurcation aneurysm (Case 7) also engaged the looping method for superselecting the incorporated, acute angle right posterior cerebral artery (PCA). A J-preshaped microcatheter was used to form the loop in the right cervical VA and advanced into the aneurysm neck. Initially the looped tip faced toward around the right PCA orifice, so the microguidewire was rotated to change direction of the microcatheter tip after placing the wire tip at the proximal half of the loop inside the microcatheter (wire steering). When the microcatheter tip was exactly directed at the right PCA, the microguidewire was advanced out of the microcatheter tip into the right PCA. Further steps for stent-assisted coiling were the same as typical procedures.

In Case 8, the right PCA was so deeply incorporated into basilar bifurcation aneurysm $\left(80^{\circ}\right)$ that it could not be separated from the aneurysm in any views. Superselecting the PCA by intraaneurysmal antegrade wire navigation was thought to be extremely difficult and at risk for aneurysm perforation. However, just the use of a C-preshaped microcatheter succeeded in directly accessing the incorporated branch with no aid of the looping method.
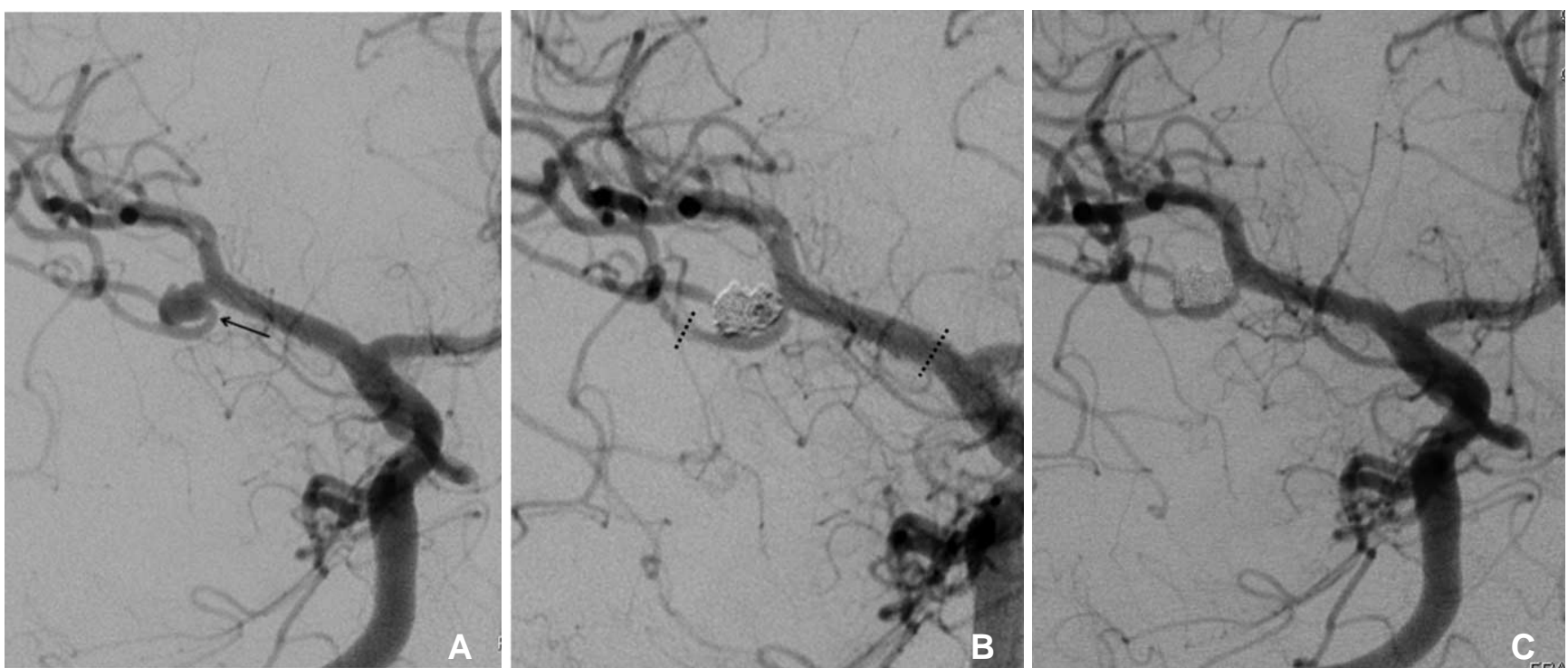

Fig. 3. A 50-year-old male with an unruptured MCA bifurcation aneurysm (Case 5), whose images are the ground of the Fig. 1B through $1 f$ illustrations.

A. Working projection shows a MCA bifurcation aneurysm with the inferior division incorporated into the sac (black arrow). The incorporated branch arises from the sac at an angle of $41^{\circ}$ relative to the $\mathrm{M} 1$ segment.

B. Completion angiogram obtained after stent-assisted coiling, whose steps are illustrated in Fig. 1., demonstrates complete occlusion of that aneurysm and normal flow in the inferior division. The stent markers are barely identified (dotted line).

C. Follow-up angiogram at 12 months shows well exclusion of that aneurysm from the circulation. 


\section{Anatomical and clinical outcomes}

Aneurysm occlusion was rated as 'total occlusion' in four cases, 'residual neck' in one, and 'residual aneurysm' in three. Thrombosis and occlusion of the PICA complicated the procedure in Case 2 with a ruptured aneurysm. This caused no neurological deficits or repeat bleeding after tirofiban administration. Aside from this complication, no procedural complication was developed in all cases. Another patient with a ruptured aneurysm (Case 4) was also free of neurological deficits at discharge. Overall, new neurological deficits were not identified in any cases.

A total of six patients underwent clinical follow-up at least one month after embolization (range, 1-12; mean, 6.3 months). Four patients had a modified Rankin Score of 0 , but the remaining two had the score of 3, which was the same as the pretreatment status of moderate disability resultant from pontine hemorrhage and rupture of another aneurysm, respectively.

Only two patients underwent conventional angiography at twelve months postembolization and proved to have aneurysms excluded from the circulation (Case 4 and 5), while only one patient followed MR angiography at 15 months with no change of a small residual neck compared to the completion angiography of the procedure (Case 7).

\section{DISCUSSION}

Complex aneurysms for endovascular treatment have diverse definitions. Many anatomical variables could be listed as being 'complex', where aneurysms with an incorporated branch are known to have high procedural difficulties and risks [3]. These aneurysms have been considered candidates for surgery rather than coiling. Recently, with advanced balloon technologies, it seems that endovascular treatment has favorable clinical results $[2,4]$. However, handling of the balloon devices requires a lot of experience and some controversies have arisen regarding the safety of the balloon remodeling technique [5]. Moreover, even a compliant balloon may not protect the incorporated branch effectively if the balloon does not herniate sufficiently into aneurysms or a good coil frame during balloon inflation collapses after balloon deflation. A multicatheter technique may be an alternative to the balloon remodeling technique in the treatment of complex aneurysms [6, 7]. However, this technique also can fail if multiple coil placements via multi-catheters cannot make a sustainable coil frame. For stent-assisted coiling, stent bridging across the aneurysm neck is not enough to protect the incorporated branch, because coils are protruded over the branch orifice toward the arterial lumen as far as stent struts stand. Therefore, it is safe to place a microcatheter in the incorporated branch before coil packing, so that coiling with stent assistance through the branch could be performed. Recently, catheter-assisted coiling also has been proposed as an alternative to the balloon remodeling or stent-assisted coiling with similar outcomes; this so called microcatheter protective technique, allegedly has some advantages over other techniques, such as easier navigation with smaller microcatheters and no need for antiplatelet medication postembolization [8]. Although BT seems more favorable to branch protection than NT, it may have its own risks as well as high failure rates. Intra-aneurysmal antegrade wire navigation toward the branch may lead to aneurysm perforation from direct wall penetration or wire jumping to the aneurysm dome. If the incorporated branch arose at an acute angle relative to the parent artery like the cases of this study, the branch access would be more difficult and riskier with this antegrade wire navigation. However, this study showed that the use of a looped microcatheter, designed to reduce the risks of the antegrade wire navigation, was all successful in accessing the incorporated, acute angle branch to proceed to BT. Although an additional, 0.014' -compatible, C- or $\mathrm{J}$-preshaped microcatheter is required for $\mathrm{BT}$, the additional expense and time for the introduction of this microcatheter seem negligible compared to the balloon remodeling or multi-catheter technique.

Even in cases with an incorporated, near right angle branch $\left(80^{\circ}\right)$, we believe that the risk of aneurysm perforation should decrease with a non-wire-guided, direct approach of a shaped microcatheter instead of intra-aneurysmal antegrade wire navigation. We don't know where the weak points are located on the aneurysm wall, and the wire tip can pierce the weak points during wire navigation. Even though the wire tip locates the branch orifice itself, the wire may jump to the aneurysm dome with pushing it in for onward advance in the branch. In contrast, if a shaped microcatheter directly approaches the branch orifice without wire navigation, the branch can be not only confirmed by contrast injection via the microcatheter, but smoothly selected by the wire with little chance of wire jumping. If the shaped mirocatheter reaches near the branch orifice, wire steering by proximal handle rotation following placement of the wire tip just inside the microcatheter tip can easily change the microcatheter direction to the branch orifice [9]. 
Considering that every incorporated branch in this study arose at an acute angle relative to the parent artery, $\mathrm{C}$ - or J-preshaped microcatheters were likely to be the best for both the direct approach and looping method. The microcatheter tip is smaller and softer and more acutely angled for these preshaped microcatheters $\left(0.014^{\prime}\right)$ as compared to the stent delivery microcatheters $\left(0.021^{\prime}\right)$ in such a way that the looping and direct approach can be more easily made for the preshaped microcatheters $\left(0.014^{\prime}\right)$.

Theoretically, the looping can fail despite following the illustrated methods: inability to make the wire looping, lack of an appropriate side branch for the resistant-branch method, or potential risks of wireinduced dissection. Although we did not experience any failure or dissection, it should be reevaluated in a large-scale study. Importantly, the angiograms should be reviewed in detail for the size and direction of the main trunk beyond the resistant branch as well as the presence of the side branches of the ICA or VA. These are key factors to success in looping.

Both surgical and endovascular treatments of complex aneurysms are challenging, even in this modern era of medicine. In some studies, endovascular treatment of complex aneurysms has yielded favorable results using new devices and techniques, though debate remains as to their risks and success rates. When it comes to wide-necked aneurysms with an incorporated, acute angle branch, BT is considered more efficient in branch preserving compared to the balloon remodeling or multi-catheter technique. However, perilous or impossible branch access represents an obstacle for ubiquitous use of this method. We suggest that the looping technique using a $\mathrm{C}$ - or J-preshaped microcatheter be a novel method for access to the incorporated, acute angle branch.

\section{References}

1. Molyneux AJ, Kerr RS, Yu LM, Clarke M, Sneade M, Yarnold JA, et al. International subarachnoid aneurysm trial (ISAT) of neurosurgical clipping versus endovascular coiling in 2143 patients with ruptured intracranial aneurysms: a randomised comparison of effects on survival, dependency, seizures, rebleeding, subgroups, and aneurysm occlusion. Lancet 2005;366:809-817

2. Lubicz B, Lefranc F, Levivier M, Dewitte O, Pirotte B, Brotchi J, et al. Endovascular treatment of intracranial aneurysms with a branch arising from the sac. AJNR Am J Neuroradiol 2006;27: 142-147

3. Jayaraman MV, Do HM, Versnick EJ, Steinberg GK, Marks MP. Morphologic assessment of middle cerebral artery aneurysms for endovascular treatment. J Stroke Cerebrovasc Dis 2007;16:52-56

4. Kim BM, Park SI, Kim DJ, Kim DI, Suh SH, Kwon TH, et al. Endovascular coil embolization of aneurysms with a branch incorporated into the sac. AJNR Am J Neuroradiol 2010;31:145151

5. Sluzewski M, van Rooij WJ, Beute GN, Nijssen PC. Balloonassisted coil embolization of intracranial aneurysms: incidence, complications, and angiography results. J Neurosurg 2006;105: 396-399

6. Kwon OK, Kim SH, Kwon BJ, Kang HS, Kim JH, Oh CW, et al. Endovascular treatment of wide-necked aneurysms by using two microcatheters: techniques and outcomes in 25 patients. AJNR Am J Neuroradiol 2005;26:894-900

7. Kwon OK, Kim SH, Oh CW, Han MH, Kang HS, Kwon BJ, et al. Embolization of wide-necked aneurysms with using three or more microcatheters. Acta Neurochir (Wien) 2006;148:1139-1145

8. Lee JY, Seo JH, Cho YD, Kang HS, Han MH. Endovascular treatment of wide-neck intracranial aneurysms using a microcatheter protective technique: results and outcomes in 75 aneurysms. AJNR Am J Neuroradiol 2011;32:917-922

9. Kwon BJ, Im SH, Park JC, Cho YD, Kang HS, Kim JE, et al. Shaping and navigating methods of microcatheters for endovascular treatment of paraclinoid aneurysms. Neurosurgery 2010;67:3440 